\title{
Análisis del Seguimiento del Punto de Máxima Potencia Global (GMPPT) con Perflles de \\ Sombras para una Granja Fotovoltaica
}

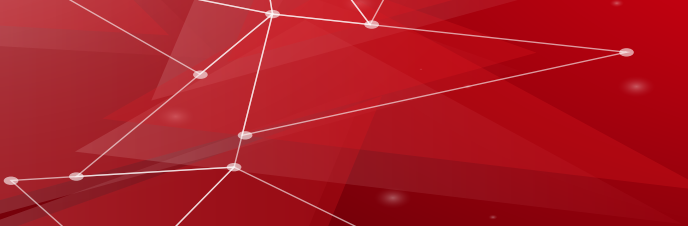

Danilo Cherres Argüello, Marcelo Pozo, Carlos Gallardo Facultad de Ingeniería Eléctrica, Escuela Politécnica Nacional

Av. Ladrón de Guevara 253

Quito, Ecuador

\section{RESUMEN}

En el presente artículo se desarrolla el análisis de un sistema para el seguimiento del punto de potencia máxima global (GMPPT) de una granja fotovoltaica (FV). El sistema a simular es un array de paneles solares conectados a un convertidor CC/CC. El software usado para este propósito en Matlab Simulink. Los perfiles de irradiancia y temperatura se los toman de una fuente local (Ecuador-Pichincha-Quito-Cotocollao). Los resultados obtenidos con la simulación permiten comprobar el buen desempeño del sistema propuesto y la ventaja de utilizar el método GMPPT.

Palabras Clave: GMPPT, photovoltaic farm, Disturbance and Observation. 


\section{Introduction}

En la actualidad la tendencia mundial es la búsqueda de nuevos suministros de energía renovable, para ello se han logrado implementar innumerables sistemas de generación de los cuales destacan la generación hidráulica, térmica, eólica, fotovoltaica, entre otras (World energy outlook. Energy access. 2016).

Con la implementación de fuentes renovables limpias se reconoce el término generación distribuida, la cual consiste en la generación de energía eléctrica mediante muchas pequeñas fuentes de generación, instaladas cerca del consumidor que se conecta a la red de distribución de energía eléctrica. Tener distribuida la generación reduce pérdidas en la red y descarga la red de transporte, esto da como resultado que ecuador genere normativas que incentiven al uso de energías renovables, sin embargo uno de los principal inconvenientes como mencionan en (Espinoza Trejo et al, 2011) una de las principales desventajas es la elevada inversión inicial requerida para la adquisición de esta tecnología.

En el presente artículo se detalla el control y simulación de un seguidor de punto de máxima potencia global (GMPPT) con perfiles de sombras para una granja fotovoltaica mediante el método de escaneamiento global. En este contexto, bajo condiciones de irradiancia uniforme, los módulos fotovoltaicos (MFV) presentan una curva potencia-voltaje en la cual se observa un máximo global, conocido como Punto de Máxima Potencia (PMP) (Kakosimos et al, 2013).

El propósito de los Seguidores del Punto de Máxima Potencia (SPMP) es operar a los MFV en el PMP, y así transferir la máxima potencia a la carga. Los módulos fotovoltaicos requieren mecanismos de rastreo del punto de máxima potencia MPPT Maximum Power Point Tracking (Rastreo Máximo del Punto de Potencia) para maximizar su eficiencia en diferentes condiciones de operación, este fenómeno se lo puede divisar en las gráficas de potencia - voltaje, al contar con varias generadoras de energía se implementa un método de GMPPT en cual maximiza la potencia global, es posible que, debido a la combinación de irradiación solar y temperatura, se obtenga una gráfica P-V con diferentes máximos. En este caso habrá distintos máximos parciales, pero solo uno global, llamado "Global Maximum Power Point". 
Actualmente diferentes técnicas de control para el seguimiento del punto de potencia máxima MPPT se presentan en los diferentes artículos, tal es el caso del método perturbar y observar (P\&O) (K. H. Hussein et al, 2010), (Boualem Bendib et al, 2015), (N. Femia et al, 2005) y (R. Khanaki et al, 2013), conductancia incremental (IncCond) (Espinoza-Trejo et al, 2014) y (Q. Mei et al, 2011), MPPT con lógica difusa (FL) (Q. Mei et al, 2011) y (S. Zahra Mirbagheri et al, 2013), redes neuronales artificiales (ANN) (M. Z. Alabedin et al, 2011), basados en algoritmos genéticos (GA) (Tajuddin et al, 2015) y (Hadji, Seddik et al, 2011) y algunos MPPT más avanzados computacionalmente como los basados en optimización de enjambre de partículas (Particle Swarm Optimization) (Tajuddin et al, 2015) y (Abdulkadir, M. et al, 2014), optimización de colonia de hormigas (Ant Colony Optimization) (Taajuddin et al, 2015) y evolución diferencial (Differential Evolution) (Tajuddin et al, 2015) y (Ramli et al, 2015). En el caso de la técnica $P \& O$, también conocida como hill climbing, la búsqueda del punto de máxima potencia lo hace modificando el valor de voltaje o corriente. Para el problema de oscilaciones presentadas al tener un paso fijo se soluciona con el método IncCond, el cual al encontrar el punto de máxima potencia deja de oscilar hasta que se presenta un cambio en el valor de potencia.

En el caso de controles MPPT basados en técnicas como lógica difusa y redes neuronales, el desempeño de los algoritmos depende de la habilidad del diseñador o el programador para obtener un buen rendimiento (Singh, R. et al, 2014). El uso de paso variable para la búsqueda del punto de máxima potencia es una estrategia orientada a mejorar la velocidad de respuesta del sistema ante cambios bruscos de radiación solar.

En este trabajo se desarrolla el análisis e implementación de un sistema para el seguimiento del punto de potencia máxima global (GMPPT) de una granja fotovoltaica (FV), la cual, se encuentra simulada dentro del software simulink de Matlab, las variables de entrada son la irradiancia y la temperatura en grados Celsius, los cuales ingresan a un convertidor CC/ CC en el cual se implementa el algoritmo de MPPT actuando como unidad de control y conectados a un bus común de 500V.

\section{Marco teórico}

Para la transformación solar a electricidad se utiliza la celda fotovoltaica, es básicamente diodo semiconductor con su p-n expuesta a la luz solar.

El principio de funcionamiento de la placa fotovoltaica se basa en la generación de electrones libres procedentes del semiconductor y su posterior absorción en los terminales de la placa fotovoltaica, depende de las características de la onda de la luz incidente, del material semiconductor y de la temperatura en que se realiza el proceso.

\section{Modelo de la placa fotovoltaica}

En la Figura 1. se representa el modelo ideal de una placa fotovoltaica. Las ecuaciones básicas de teoría de semiconductores nos describen la Característica V-I para una celda ideal. 


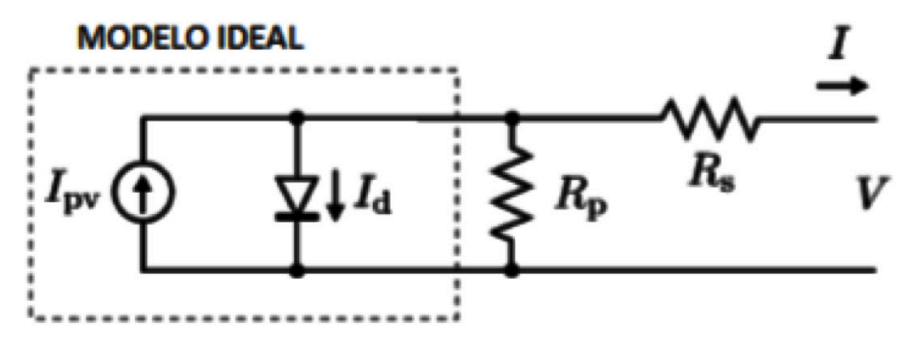

Figura 1. Modelo V-I ideal

Donde :

Ipv: corriente generada por irradiancia

Id: Corriente que circula por el diodo

Rs: Resistencia en serie

Rp: Resistencia en paralelo

V: voltaje a la salida del panel solar

I: corriente de salida del panel solar

En la práctica las placas fotovoltaicas están compuestas por un conjunto de celdas conectadas entre sí. Dependiendo del número de celdas en serie ([2]?) y de celdas en paralelo ([2]? la corriente de la placa fotovoltaica es:

$$
I=I_{p v}-I_{o}\left[\exp \left(\frac{v+R_{s} I}{v_{t} a}\right)-1\right]-\frac{v+R_{s} I}{R_{v}}
$$

Donde:

K: Constante de Boltzman (J/oK)

Q: carga del electrón (C)

T: Temperatura (oK)

A: factor de idealidad del diodo

La corriente depende del voltaje térmico $(\mathrm{Vt})$ de la celda que cambia dependiendo de la temperatura real de la celda, este voltaje térmico es igual a:

$$
V_{t}=\frac{N_{s} K T}{q}
$$


Este modelo utiliza un solo diodo para representar el modelo matemático de un sistema eléctrico. Pese a que existen otros modelos que introducen otros elementos para aumentar la precisión.

\section{Curvas características del panel fotovoltaico}

Las curvas características I-V y P-V son las dos gráficas que representan el funcionamiento de los módulos fotovoltaicos. La curva característica I-V determina las posibles combinaciones de voltaje y corriente en la que puede trabajar el sistema bajo unas condiciones ambientales fijas (radiación solar y temperatura ambiente) (Cabrera-Tobar et al. 2019).

Una de las características más interesantes de los módulos solares es la posibilidad de trabajar tanto en fuente de tensión como de corriente. Dependiendo de los valores I-V, sabemos que el funcionamiento a la izquierda del MPP es de fuente de corriente. Mientras que los valores a la derecha trabajaran como fuente de tensión.

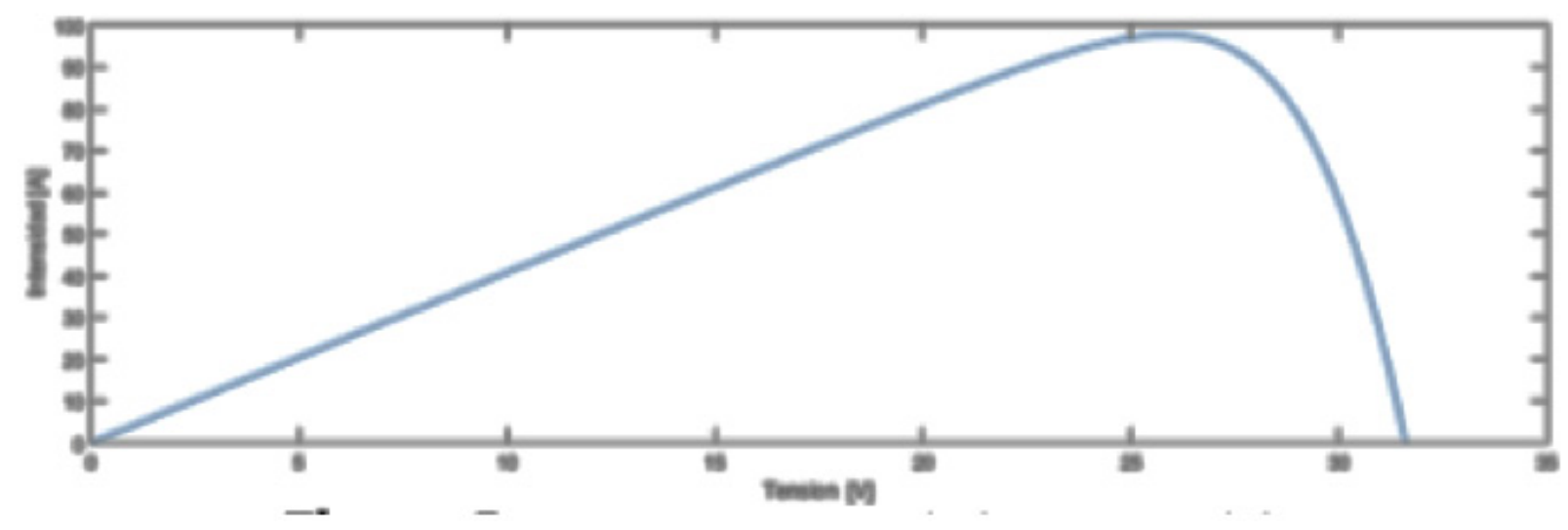

Figura 2. Diagrama potencia - Tensión

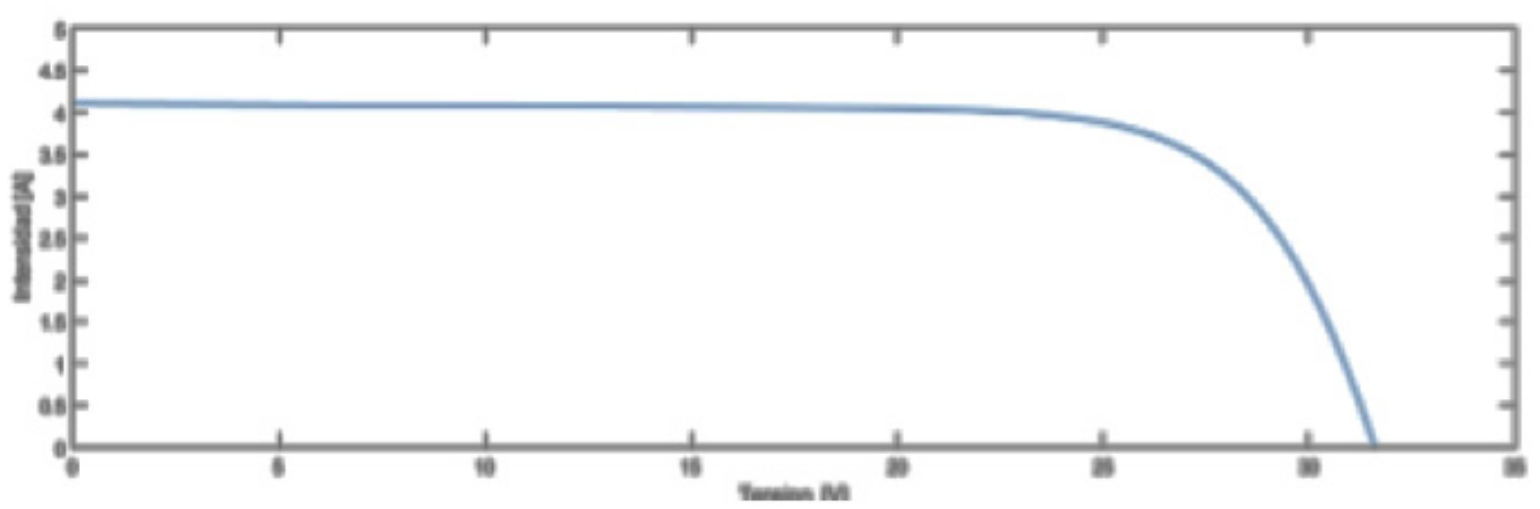

Figura 3. Diagrama Intensidad - Tensión

Es posible que, debido a la combinación de irradiación solar y temperatura, se obtenga una gráfica P-V con diferentes máximos. En este caso habrá distintos máximos parciales, pero solo uno global, llamado "Global Maximum Power Point". 


\section{Análisis de Curvas}

El análisis se realiza mediante la variación independiente de tres parámetros: irradiancia, temperatura y resistencia en serie. La Figura 4 muestra el diagrama Potencia (?)) y Tensión (?)), variando los valores de Irradiancia (??/?2). La Figura 5 muestra el diagrama Potencia (??) y Tensión (?), variando los valores de Temperatura (⁇?).

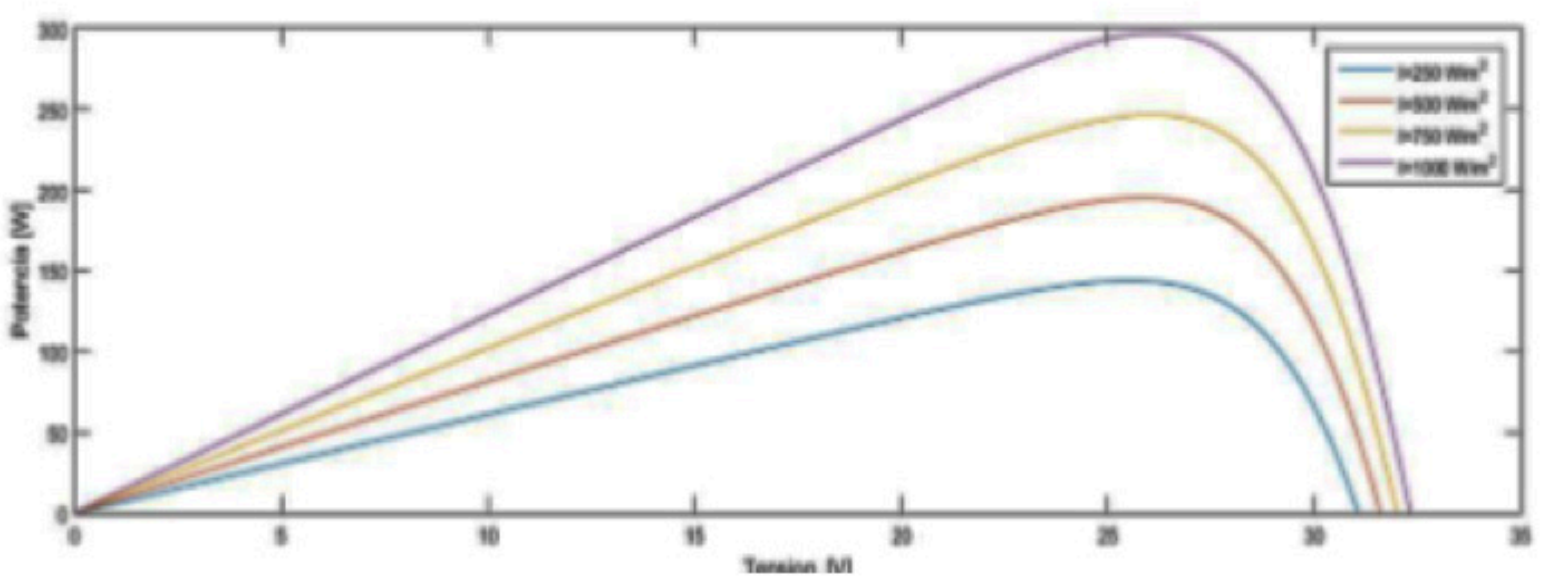

Figura 4. Diagrama $P$ - V

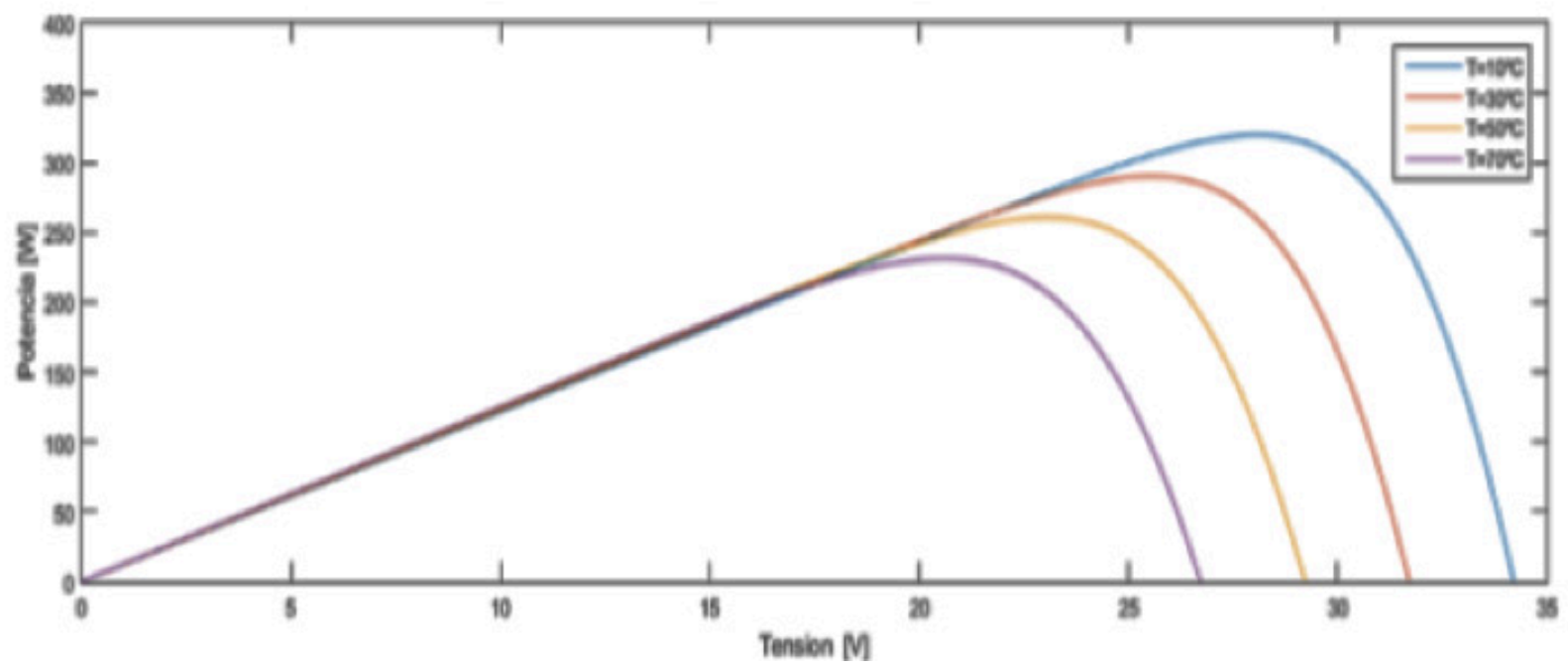

Figura 5. Diagrama $P-V$

\section{Métodos MPPT}

Maxium Power Point Trackers (MPPT) (en español: seguidores de puntos de máxima potencia) son algoritmos que se encargan de maximizar la potencia de salida de los módulos fotovoltaicos. La conexión directa de placas solar a una carga fija lleva a un punto de trabajo determinado por la intersección de las características I-V de los dos elementos. Por lo que, en general, este punto no coincide con el MPP del panel, restando así eficiencia al sistema fotovoltaico.

Los MPPT utilizan, mediante un controlador estático, diversos algoritmos que se encargan de, independientemente de la radiación y la temperatura, buscar el voltaje Vmpp y la corriente Impp que produzcan la máxima potencia de salida Pmpp. 
En la literatura existen numerosas técnicas de seguimiento del punto de máxima potencia (MPPT):

-Perturbar y observar.

-Hill-Climbing.

-Lógica Difusa.

-Control de correlación de la ondulación (RCC).

-Conductancia incremental.

-Control de seguimiento extremo (ESC, por sus siglas en inglés).

Algunos presentan un mejor rendimiento en estado estacionario, mientras que otros son superiores durante las transiciones.

\section{- $\quad$ Perturb and Observe (P\&O)}

El algoritmo se puede observar en la Figura 9, dicho método es muy utilizado debido a su simplicidad de funcionamiento, este consiste en modificar el ciclo útil de un convertidor de potencia conectado a la salida del arreglo fotovoltaico, con el cambio de ciclo útil se modifica la corriente extraída del arreglo fotovoltaico, por lo consiguiente la potencia obtenida se modifica, esto en base a la medición de la potencia y realizando una comparación entra la potencia actual (pact) que se tiene y la potencia anteriormente obtenida (pant), de esta 4 manera dependiendo el valor de la comparación se decide el valor o dirección de la modificación del ciclo útil (pert).(Ruiz, C. et al, 2010)

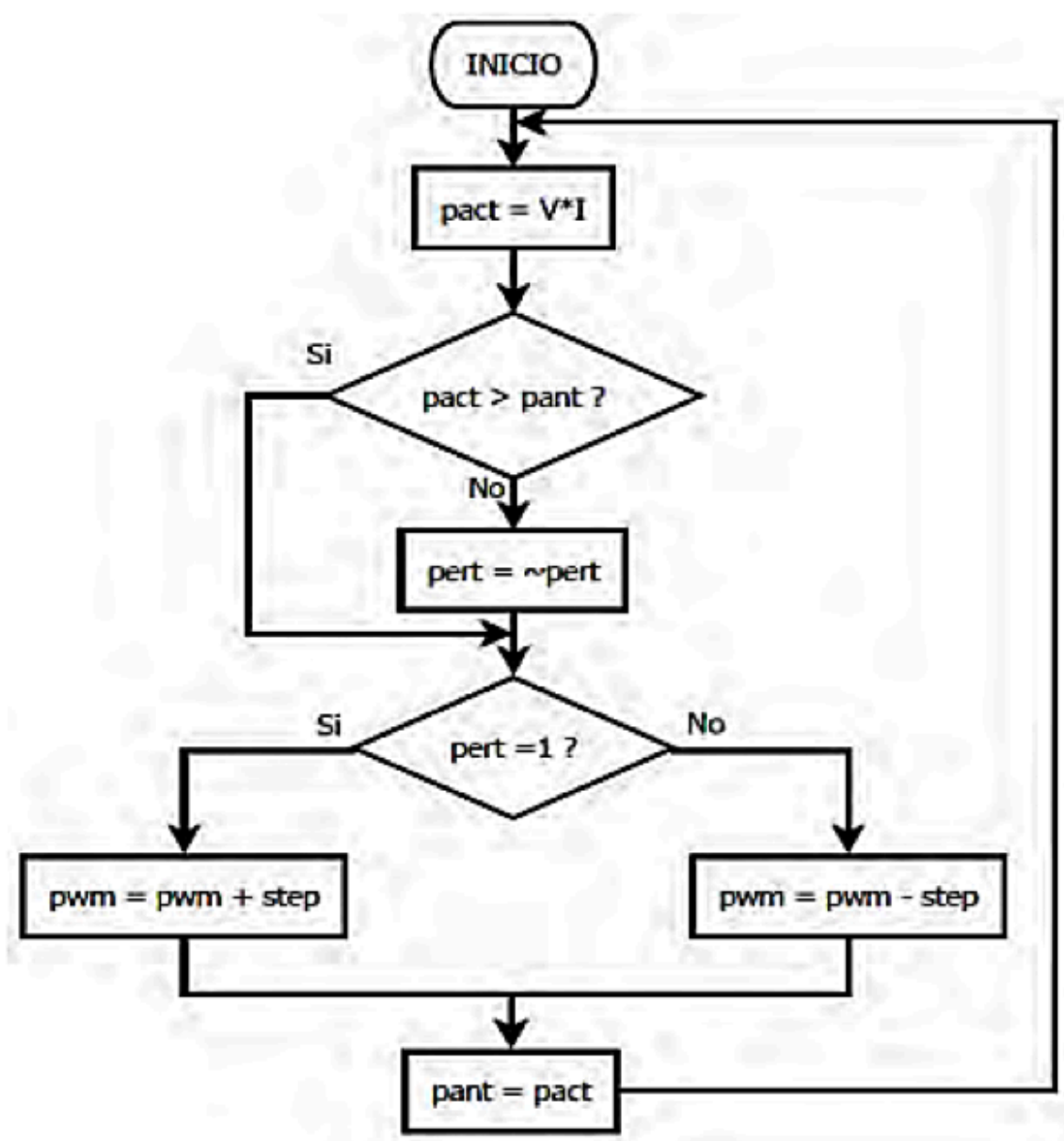

Figura 6. Algoritmo Perturbar y Observar 
El funcionamiento de este algoritmo consiste en si el voltaje de operación se perturba en cierta dirección y la potencia extraída del mismo se incrementa significa que el punto de operación se ha movido hacia el MPP, por lo tanto el voltaje de operación deberá perturbarse en la misma dirección, o si la potencia extraída del arreglo fotovoltaico disminuye, el punto de operación se ha movido en dirección opuesta de la ubicación del MPP, por consiguiente el voltaje de operación deberá ser perturbado en dirección contraria a la que se tenía.

\section{- $\quad$ Algoritmo Hill-Climbing}

Es muy similar al Perturbar y Observar, de manera que comienza con una solución arbitraria e intentando encontrar una mejor solución en donde se varía incrementalmente un único elemento de la solución. Si el cambio produce una mejor solución, otro cambio incremental se le realizará a la nueva solución, repitiendo este proceso hasta que no se pueda encontrar mejoras. Otra forma de describir el funcionamiento del algoritmo es de que Hill-Climbing intenta maximizar (o minimizar) una función objetivo. En cada iteración, el algoritmo ajustará un único elemento y determinará si el cambio mejora el valor de la función, Cuando se presentan puntos globales y locales en el sistema, el algoritmo Hill Climbing solo podrá converger al máximo local, de tal manera que pare mejorar la eficiencia de posicionamiento se suele corregir el algoritmo.(Khanna, R. et al, 2014)

\section{- $\quad$ MPPT con sombreado parcial}

Este tipo de métodos funciona muy bien ya sea con un sólo panel solar que regularmente su uso es para casas o con varios conectados en serie o paralelo para uso industrial en el cual la potencia demandada es mucho mayor. Sin embargo, el mayor problema por el cual no se quieren usar los paneles fotovoltaicos, es por la variación en su eficiencia que estos presentan, en donde como ya se mencionó, los paneles dependen de la radiación solar, haciendo que las nubes o cualquier objeto que genere sombrado sobre el panel sea una perturbación hacia nuestro sistema. Para este tipo de perturbaciones un simple MPPT no es suficiente, las soluciones varían, pero no deja de ser un arreglo que se tiene que hacer sobre el algoritmo de MPPT, para poder reconocer cuando se presenten estas variaciones y poder actuar de forma inmediata. En la Figura 10 se puede visualizar cuando los paneles están bajo un sombreado parcial.
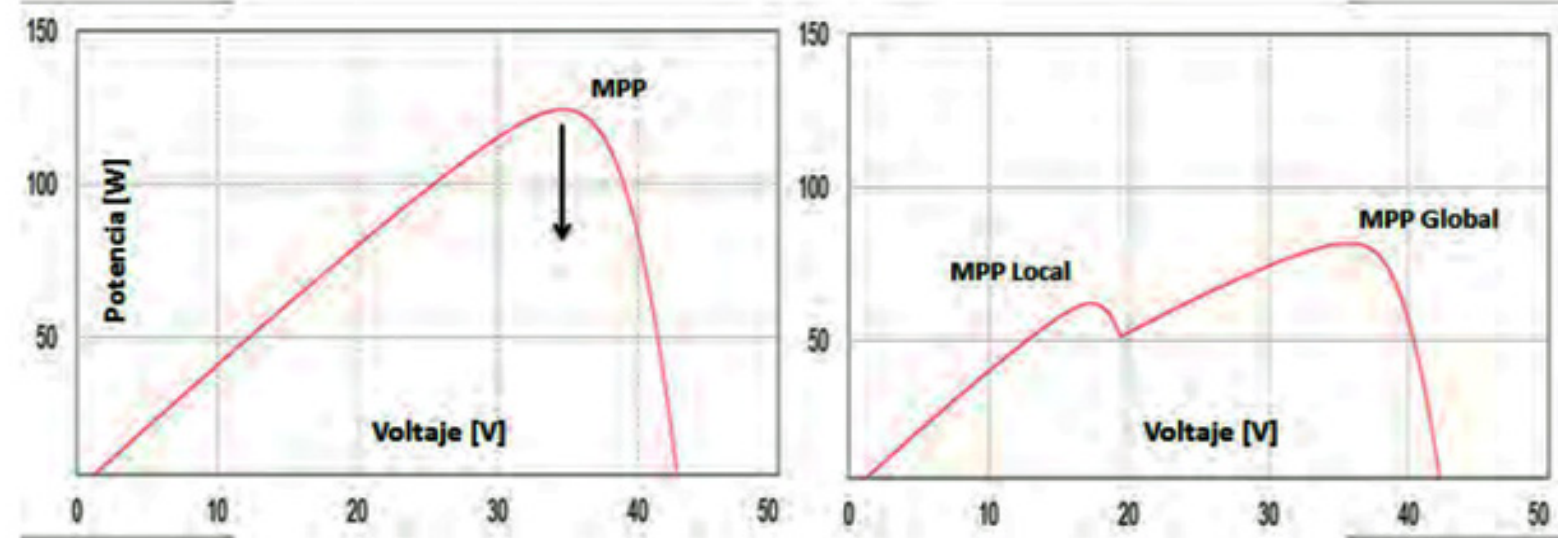

Figura 7. Curva característica con sombreado mostrando MPP Global y Local 
Bajo estas condiciones de sombreado, las curvas características presentan variaciones en el MPP, teniendo un MPP global y otro local, siendo el global el máximo de ellos, pero dependiendo la cantidad del panel que se encuentra sombreado y el panel con sombreado se pueden observar los siguientes dos casos mostrados en la Figura 11

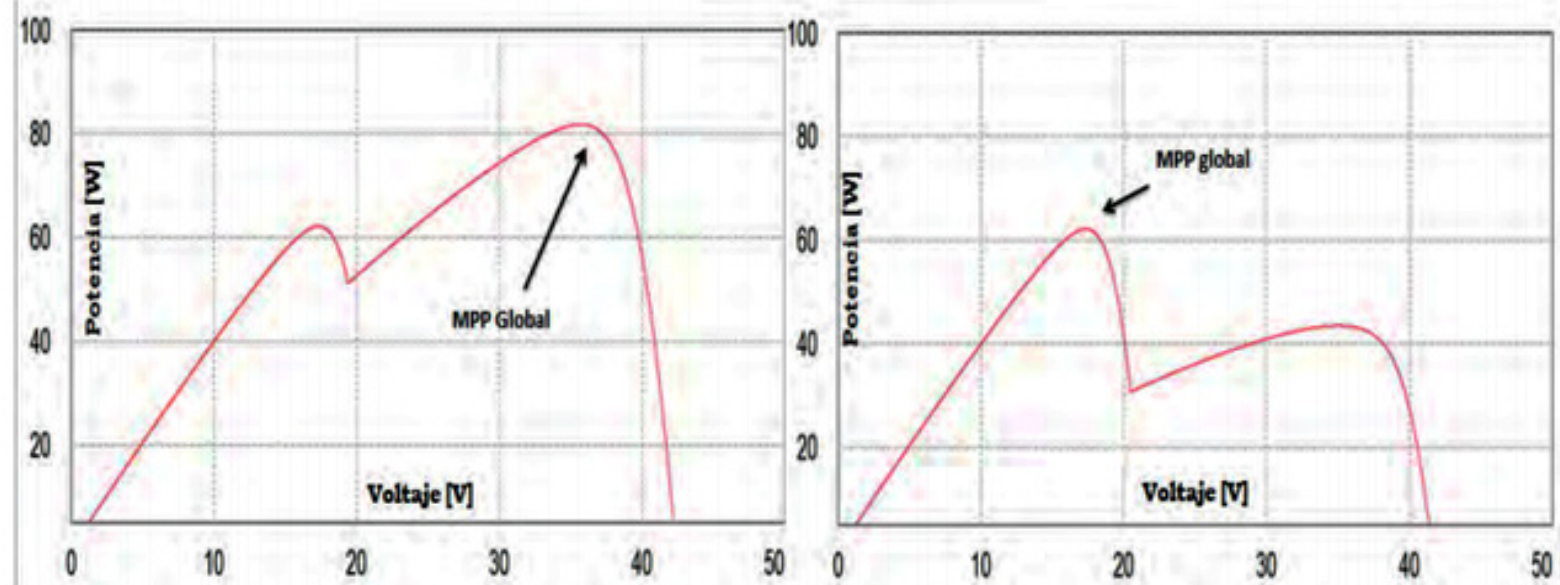

Figura 8. Diferencias entre variaciones de sombreado parcial en paneles solares

El algoritmo de MPPT en el trabajo (Liu, G. et al, 2011)utiliza el método de la perturbación y observación, se modificó para considerar que el panel solar se encuentra en condiciones de sombreado parcial, realizando comparaciones entre las potencias y voltajes actuales (Potencia_actual y Voltaje_actual) medidas en el arreglo fotovoltaico, contra las tomadas un muestreo anterior (Potencia_ant y Voltaje_anterior) y en el caso de tener sombreado parcial realizar una acción específica.

En el trabajo dispuesto en (Femia, N. et al, 2008) toma MPPT individuales para cada panel solar y así obtener el punto de máxima potencia para cada módulo fotovoltaico puede ser asociado a un convertidor DC / DC para extraer toda la potencia., denominándola seguimiento distribuido del punto de máxima potencia (DMPPT), como se muestra en la figura 12.

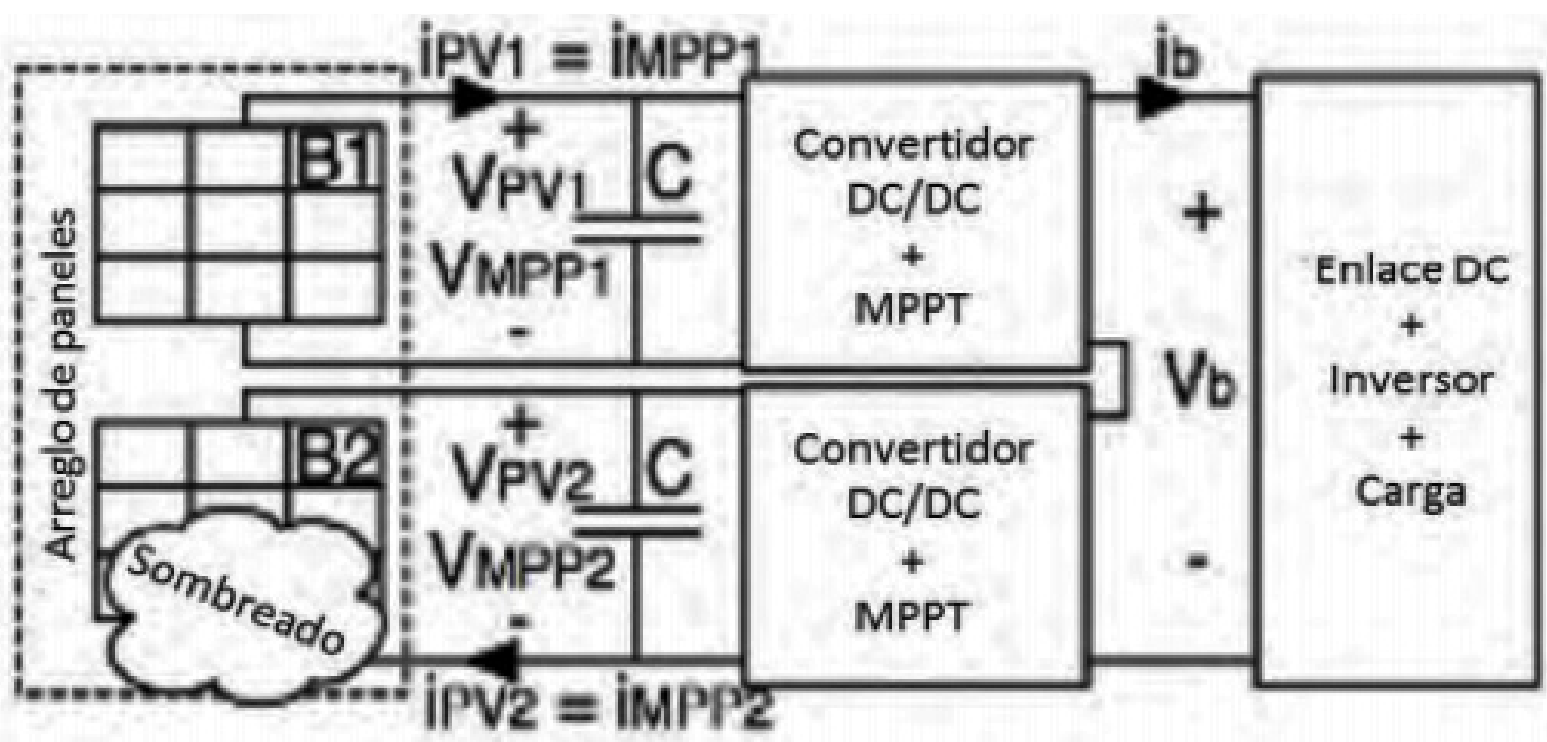

Figura 9. Configuración individual de MPPT's para paneles en donde a cada panel PV se aplica un control $M P P T$ con convertidor $D C / D C$ 
En el trabajo (Peng L. et al, 2011) se presenta un control por inversores Flyback controlado por sensores activos para el seguimiento de punto de máxima potencia (MPPT) para una pequeña instalación fotovoltaica (PV). Cuando un sistema de energía fotovoltaica se construye con un número de sistemas de energía pequeñas, el coste total del sistema se incrementará y será un asunto de preocupación. Para superar esta dificultad, en este trabajo se propone un sistema fotovoltaico que no utiliza el sensor de corriente caro, pero utiliza el método de estimación de la corriente fotovoltaica a partir del voltaje PV.

Para el trabajo propuesto por (A. W. Leedy, L. et al, 2012) Control de seguimiento extremo (ESC) hace frente a las características multimodales de campo fotovoltaico con sombreado variables, este estudio propone una estrategia de control global MPPT basada en ESC secuencial, basado en modelado aproximado y análisis de las características bajo circunstancias de sombreado variable.

El Control por modos deslizantes es sinónimo de robustez, buena respuesta dinámica, estabilidad ante variaciones grandes de la carga y de la fuente, son algunas de las características que presentan los sistemas al ser retroalimentados cuando se les aplica un control por modos deslizantes, el control se basa, a partir de la definición de una superficie atractora denominada de deslizamiento que "atrae" a las trayectorias de los estados del sistema hacia ella y hace que dichos estados del sistema se mantengan ahí, llevando al sistema al punto de operación deseado una vez que se llega a la superficie definida, tal proyecto se define en (Utkin et al, 2016).

\section{Procedimiento}

Se toma el modelo power_4PVarray_400kW, disponible en la librería de Simulink Matlab, el cual muestra un modelo promedio de un pequeño parque fotovoltaico (400 kW) conectada a una red de $25 \mathrm{kV}$ utilizando un convertidor de dos etapas como se muestra en la Figura 13.

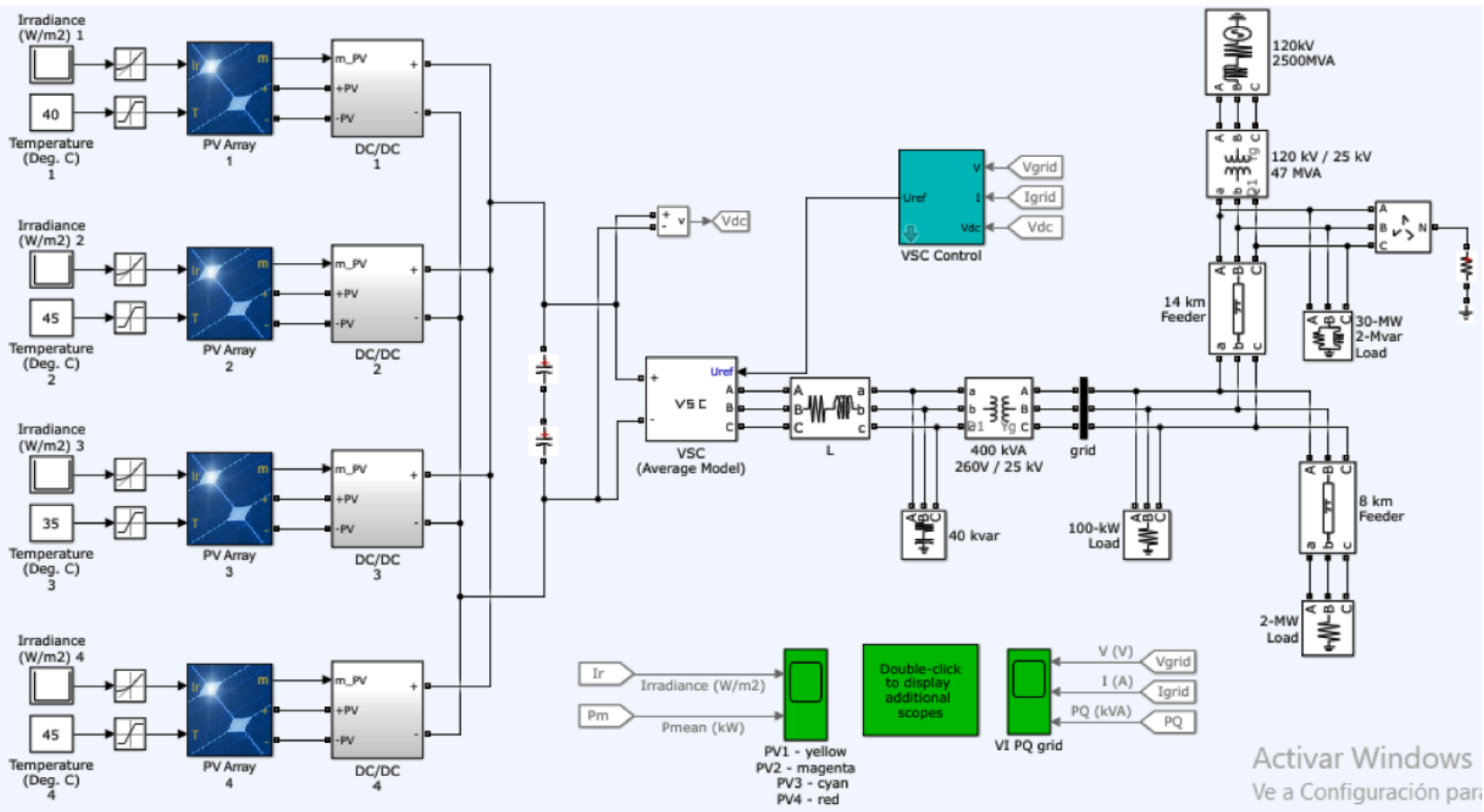


Las variables de entrada para el sistema son la irradiancia y la temperatura, para esto, se modifica el modelo generando un código .m, el cual pide el mes y dia del análisis del año 2018, estos datos son sacados de la base de datos de la Secretaría de Ambiente del Municipio del Distrito Metropolitano Quito.

El parque fotovoltaico consta de cuatro matrices fotovoltaicas que suministran cada una un máximo de 100 kW a 1000 W / m2 de radiación solar, un solo bloque de matriz PV consta de 64 cadenas paralelas donde cada cadena tiene 5 módulos SunPower SPR-315E conectados en serie.

\section{Convertidor DC/DC}

Cada grupo fotovoltaico está conectado a un convertidor DC / DC. Las salidas de los convertidores de refuerzo están conectadas a un bus de CC común de 500 V. Cada impulso se controla mediante los Perseguidores de puntos de potencia máxima individuales (MPPT). Los MPPT utilizan la técnica de "Perturbación y Observación" para variar el voltaje a través de los terminales de la matriz FV para obtener la máxima potencia posible.

\section{Convertidores y transformadores}

Un convertidor de fuente de voltaje trifásico (VSC) convierte los 500 V CC a 260 V CA y mantiene el factor de potencia de la unidad. Se utiliza un transformador de acoplamiento trifásico de 400 kVA $260 \mathrm{~V} / 25$ kV para conectar el convertidor a la red. El modelo de cuadrícula consta de alimentadores de distribución típicos de 25 kV.

\section{Cargas}

Las Cargas del sistema son muy variadas, desde cargas simples como 100kW directamente conectadas a las líneas, así como sistemas distancia de 14 kilómetros, cargas de $120 \mathrm{~kW} / 2500 \mathrm{MVA}$, de 30MW a 2MVAR.

\section{Control MPPT}

Para el control MPPT se desarrolla un script en .m (Figura 14) esto permite tener una noción más clara del control implementado, dentro de la programación se puede observar que, se inicia leyendo las perturbaciones dispuestas por el modelo y se realiza una comparación utilizado la fórmula de $\mathrm{P}=\mathrm{V}^{\star} \mathrm{I}$ ya que utiliza el algoritmo de perturbación y observación. 

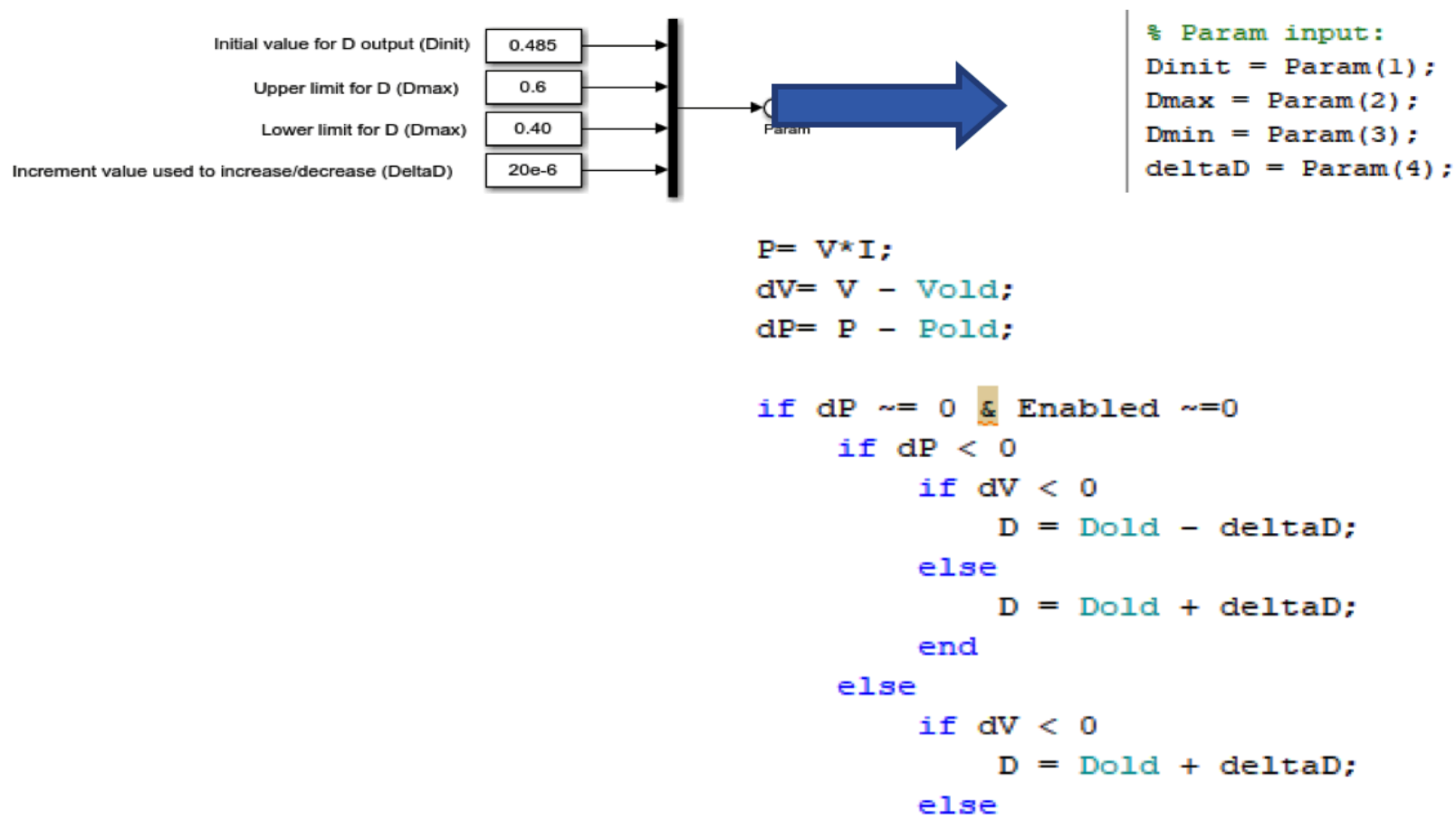

Figura 11. Desarrollo del script en .m

\section{Análisis y resultados}

Para los análisis se toman los datos suministrados por la Secretaría de Ambiente del Municipio del Distrito Metropolitano Quito, los cuales se sacan en formato .xlsx. En la figura 12 se muestran los datos de radiación solar y potencia de salida.
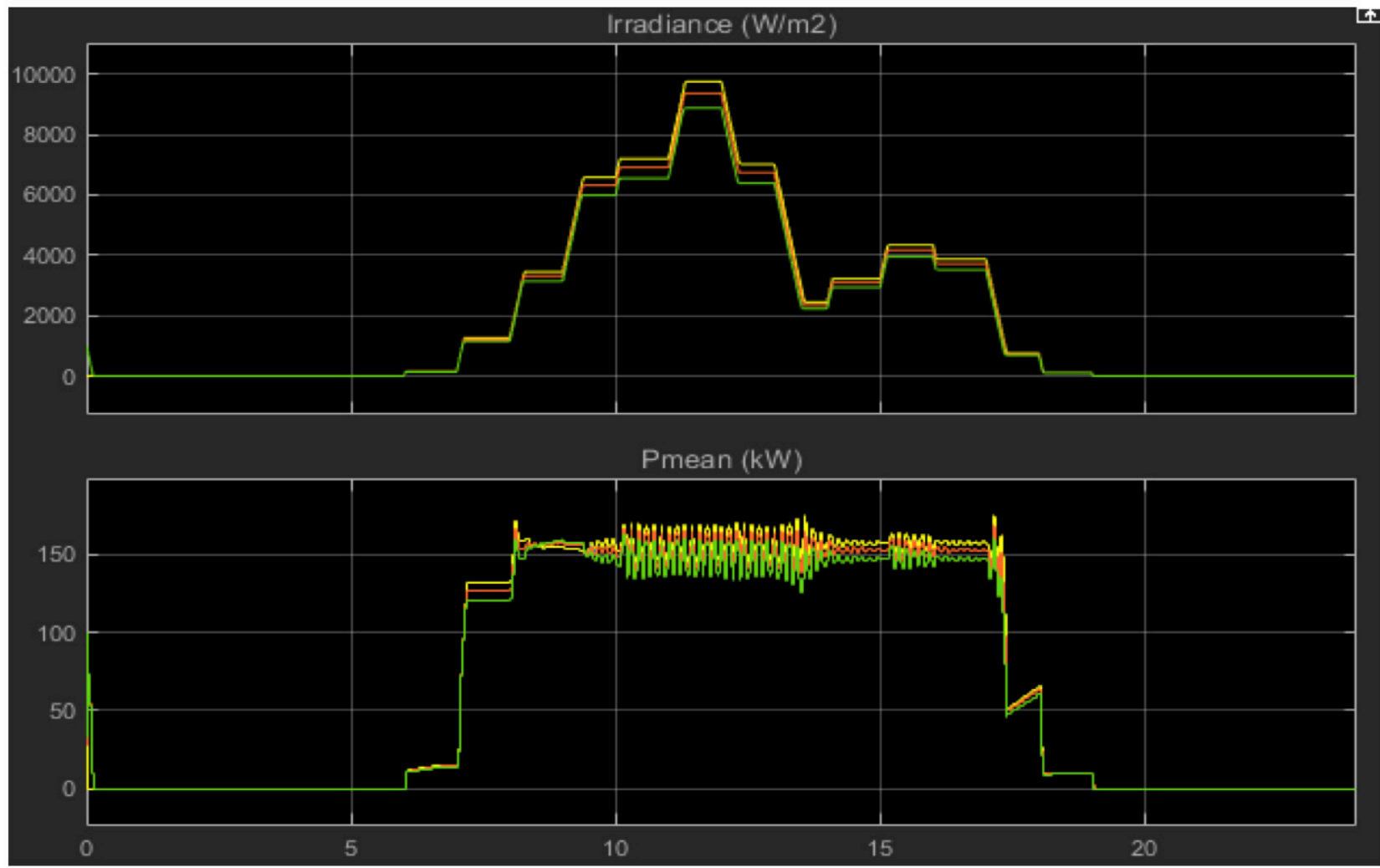
Ahora se ingresan los datos con presencia de sombra en los cuatro paneles, para poder visualizar la acción del GMPPT se ingresan los datos de la Tabla 1.

\begin{tabular}{|c|c|}
\hline Fecha & 9 de marzo \\
\hline Efecto de sombra & si \\
\hline Numero de paneles (con sombra) & 4 \\
\hline \multicolumn{2}{|l|}{ Panel 1} \\
\hline Sombreado: & $20 \%$ \\
\hline Inicio: & $6 h 00$ \\
\hline Fin: & $17 \mathrm{~h} 00$ \\
\hline \multicolumn{2}{|l|}{ Panel 2} \\
\hline Sombreado: & Completamente \\
\hline Inicio: & $8 \mathrm{~h} 00$ \\
\hline Fin: & $17 \mathrm{~h} 00$ \\
\hline \multicolumn{2}{|l|}{ Panel 3} \\
\hline Sombreado: & $60 \%$ \\
\hline Inicio: & $11 \mathrm{~h} 00$ \\
\hline Fin: & $18 \mathrm{~h} 00$ \\
\hline \multicolumn{2}{|l|}{ Panel 4} \\
\hline Sombreado: & Completamente \\
\hline Inicio: & $8 \mathrm{~h} 00$ \\
\hline Fin: & $15 \mathrm{~h} 00$ \\
\hline
\end{tabular}

Tabla 1. Valores de sombras en los paneles

En la Figura 16 se puede observar las variaciones de irradiancia y potencia que se obtiene a partir de la presencia de sombra establecida en la Tabla 1.

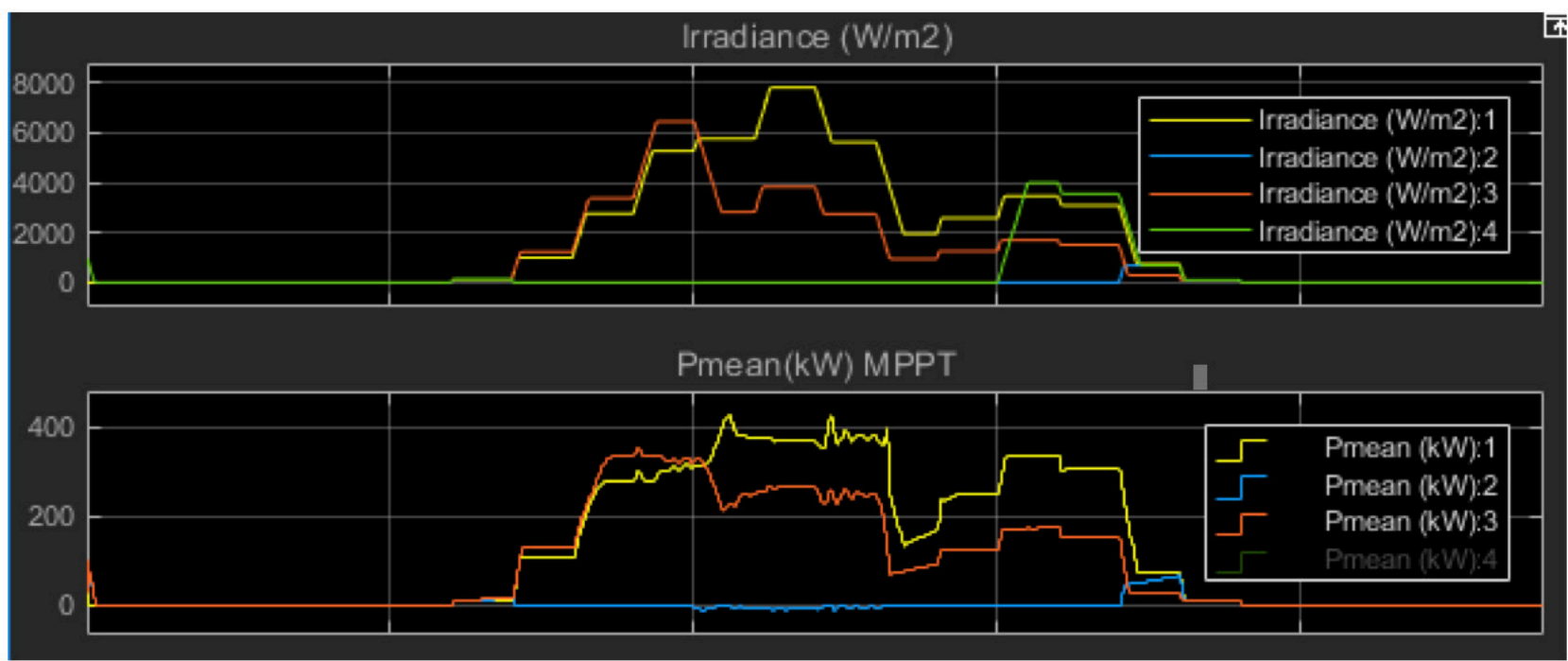

Figura 13. Valores de Potencia e irradiancia con efecto Sombra 
Ahora se procede a implementar un archivo .m el cual permite obtener lo valores máximos de potencia global como se muestra en la Figura 17.

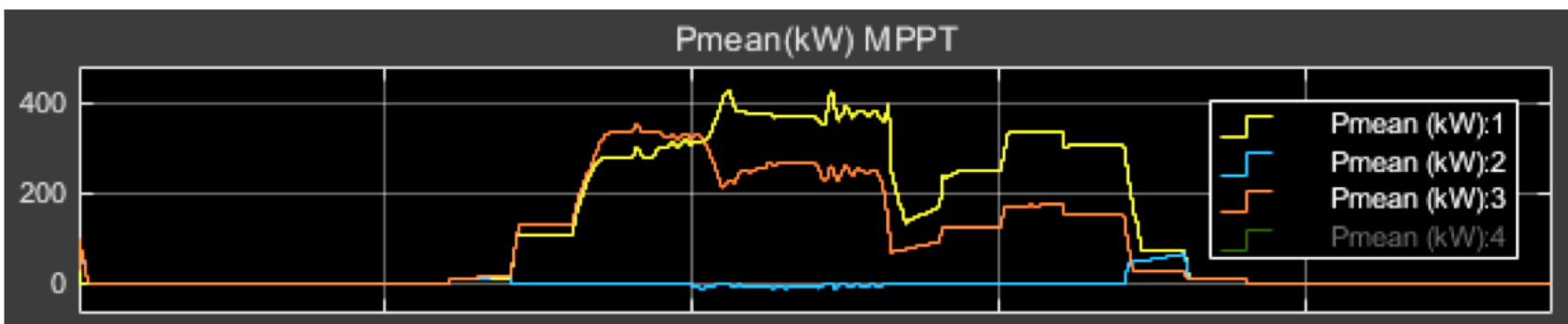

Pmean(kW) GMPPT

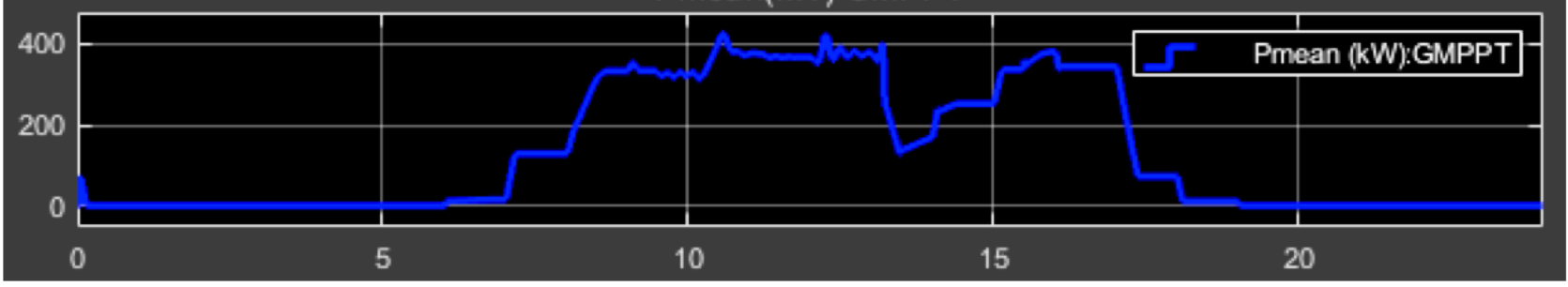

Figura 14. Utilización de GMPPT

De los resultados, se puede observar que, con el control implementado, la potencia activa resultante de los paneles solares sobrepasa el $20 \%$ cuando se considera un control normal del MPPT.

\section{CONCLUSIONES}

-La utilización del GMPPT permite aprovechar la máxima potencia existente en los paneles solares como se muestran en los resultados, sin embargo, se nota que los datos son muy grotescos, por tal motivo se recomienda que para análisis posteriores se considere un procesador más robusto y así simular de todo un dia cada 10 minutos o menos.

-La variación de irradiancia es directamente proporcional a la cantidad de corriente producida.

-Con la variación de la irradiancia el MPP vemos que varía muy poco su valor en comparación a la gran cantidad reducción de potencia aportada.

-Al variar la temperatura manteniendo la irradiancia fija la temperatura es inversamente proporcional al voltaje y la corriente no varía.

-En la variación de la temperatura las variaciones de los valores de MPP son menores, se nota que la variación importante se da en el valor de que se desplaza de manera horizontal según aumenta o disminuye la temperatura.

-La relación de la resistencia ósea la calidad de la placa afecta directamente a la potencia entregada, este parámetro se obtiene del ????]??h???? del fabricante de la placa.

-El modelo existente dentro de Matlab simulik cumple con las condiciones necesarias para su análisis, contando con parámetros de entrada, control y cargas eléctricas. 
-Dentro del control mediante MPPT al realizarse en un archivo .m se facilita ver el algoritmo implementado para posteriormente ser modificado basándose en un sistema más robusto.

\section{REFERENCIAS}

A. W. Leedy, L. Guo, K.A. Aganah. 2012. "A Constant Voltage MPPT Method for a Solar Powered Boost Converter with DC Motor Load." ISBN - IEEE: 978-1-4673-13759/12.

Abdulkadir, M.; Yatim, A. H. M.; Yusuf, S. T. 2014. "An Improved PSObased MPPT Control Strategy for Photovoltaic Systems." International Journal of Photoenergy.

Boualem Bendib, Hocine Belmili, Fateh Krim. 2015. "A Survey of the Most Used MPPT Methods: Conventional and Advanced Algorithms Applied for Photovoltaic Systems." Renewable and Sustainable Energy Reviews 45: 637-648.

Cabrera-Tobar, Ana et al. 2019. "The Effect of Ambient Temperature on the Yield of a 3 MWp PV Plant Installed in Ecuador." In Proceedings - 2019 IEEE International Conference on Environment and Electrical Engineering and 2019 IEEE Industrial and Commercial Power Systems Europe, EEEIC/I and CPS Europe 2019, Institute of Electrical and Electronics Engineers Inc.

Espinoza-Trejo, D. R., Bárcenas, E., Compeán, I., Campos-Delgado, D. U., \& PecinaSánchez, J. A. 2014. "Evaluación Experimental de Un Seguidor Del Punto de Máxima Potencia En Sistemas FV Basado En Un Control Por Linealización Entrada-Salida Con Aplicaciones a Micro-Redes de CD." XVI Congreso Latino Americano de Control Automático: 14-17.

Femia, N.; Lisi, G.; Petrone, G.; Spagnuolo, G.; Vitelli, M. 2008. "Distributed Maximum Power Point Tracking of Photovoltaic Arrays: Novel Approach and System Analysis." Industrial Electronics, IEEE Transactions on 55(7): 2610,2621.

HADJI, Seddik; GAUBERT, Jean-Paul; KRIM, Fateh. 2011. "Genetic Algorithms for Maximum Power Point Tracking in Photovoltaic Systems. En Power Electronics and Applications." 14th European Conference on. IEEE: 1-9.

K. H. Hussein, Muta, T. Hoshino and M. Osakada. 2010. "Maximum Photovoltaic Power Tracking: An Algorithm for Rapidly Changing Atmospheric Conditions." IEEE Proceedings on Generation, Transmission and Distribution 142: 59-64.

Kakosimos, P. E., Kladas, A. G., and Manias, S. N. 2013. ") Fast Photovoltaic-System Voltageor Current-Oriented MPPT Employing a Predictive Digital Current Controlled Converter." IEEE Transactions on Industrial Electronics: 5673-85.

Khanna, R., Zhang, O., Stanchina. 2014. "Maximum Power Point Tracking Using Model Reference Adaptive Control." IEEE Transactions on Power Electronics: 1490-99.

Liu, G.; Wang, P.; Wang, W.; Wang, Q. 2011. "MPPT Algorithm Under Partial Shading Conditions." ", Elect. Inf. Eng. And Mech 1: 91,98. 
M. Z. Alabedin, E. F. El-Saadany, and M. M. A. Salama. 2011. "Maximum Power Point Tracking for Photovoltaic Systems Using Fuzzy Logic and Artificial Neural Networks." Power and Energy Society General Meeting: 1 -9.

N. Femia, G. Petrone, G. Spagnuolo, and M. Vitelli. 2005. "Optimization of Perturb and Observe Maximum Power Point Tracking Method." IEEE Transactions on Power Electronics 20(4): 963-73.

Peng L.; Yaoyu L.; Seem, J.E. 2011. "Sequential ESC-Based Global MPPT Control for Photovoltaic Array With Variable Shading." IEEE Transactions 2(3): 348,358.

Q. Mei, M. Shan, L. Liu and J. M. Guerrero. 2011. "A Novel Improved Variable StepSize Incremental-Resistance MPPT Method for PV Systems." IEEE Transactions on Industrial Electronics 58(6): 2427-34.

R. Khanaki, M. A. M. Radzi, M. H. Marhaban. 2013. "Comparison of ANN and P\&O MPPT Methods for PV Applications under Changing Solar Irradiation." Clean Energy and Technology (CEAT) IEEE Conference: 287 - 292.

RAMLI, Makbul AM. 2015. "A Modified Differential Evolution Based Maximum Power Point Tracker for Photovoltaic System under Partial Shading Condition." Energy and Buildings 103: 175-84.

Ruiz, C.; Beristáin, J.; lan, M. 2010. "Estudio Del Algoritmo de Seguimiento de Punto de Máxima Potencia Perturbar y Observar." Revista de ingeniería eléctrica y computación 8: 17-23.

S. Zahra Mirbagheri, S Mekhilef, S. M. Mirhassani. 2013. "Inc.Cond Method Using Conventional Interleaved Boost Converter." Mediterranean Green Energy Forum 42(13): 24-32.

SINGH, R.; RAJPUROHIT, B. S. 2014. "Performance Evaluation of Grid Connected Solar Photovoltaic (SPV) System with Different MPPT Controllers." Energy Integration. Springer Singapore: 97-124.

Tajuddin, M. F. N. 2015. "Perturbative Methods for Maximum Power Point Tracking (MPPT) of Photovoltaic (PV) Systems." International Journal of Energy Research.

Utkin, V. I. 2016. "Sliding Modes and Their Application in Variable Structure Systems." MIR Publishers 5(7).

World Energy Outlook. Energy Access. 2016. http://www.worldenergyoutlook.org/media/ weowebsite/energymodel/documentation/WEO2016_Chapter02_acces.pdf (June 11, 2017). 